\title{
Vorwort zur 1. amerikanischen Ausgabe
}

Untersuchung und Lehre der Korrosion haben im MIT (Massachusetts Institute of Technology) eine große Tradition. Professor W. R. WhItney, der später der erste Direktor der „Research for the General Electric Company“ wurde; begründete diese Tradition mit seiner klassischen Abhandlung über die Korrosion von Eisen, die $1903 \mathrm{im}$,Journal of the American Chemical Society" veröffentlicht wurde. Dieser Artikel regte zu den bedeutenden Korrosionsuntersuchungen an, die von Professor W. H. WaLkER und später von Professor W. WhITMaN und seinen Mitarbeitern im von WALKER gegründeten ,Laboratory of Applied Chemistry" durchgeführt worden sind. Die Korrosionslehre wurde im Jahre 1903 durch Professor WALKER mit einer Vorlesung über die chemische Beständigkeit der Werkstoffe eingeführt. Diese Vorlesung erhielt 1912 den Titel „Konstruktionswerkstoffe“ und wurde zunächst unter Mitwirkung von Professor W. K. LEwIs und später von Professor LewIs allein gehalten. Im MIT begann die Vorlesung über das Thema „Korrosion“ im Jahre 1922 und wurde von Professor Whitman gehalten. Später übernahmen diese Vorlesung verschiedene andere Mitglieder des „Department of Chemical Engineering", u. a. auch Professor LEwIs. In den Jahren von 1931 bis 1942 las Professor R. S. WILLIams vom ,Department of Metallurgy“ über „,Korrosion und hitzebeständige Legierungen". Im Jahre 1938 übernahm der Verfasser die durch Professor Whitman eingeführte systematische Vorlesung über die Korrosion. Nach einer durch den Zweiten Weltkrieg verursachten Unterbrechung wurden die Vorlesungen im „Department of Metallurgy" wieder aufgenommen und bis in die Gegenwart fortgesetzt.

Das vorliegende Buch gründet sich auf die über ein Semester gehende Vorlesung mit dem Titel „Korrosion“, welche der Verfasser für Studenten der höheren Semester und für die graduierten Studierenden am MIT vorträgt. Diese Vorlesung bezweckt, die Studenten in die wissenschaftlichen Grundlagen der Korrosion sowie in die Grundlagen der Korrosionsschutztechnik einzuführen.

In den Vorlesungen beschäftigen wir uns also mit den Fragen, warum die Metalle korrodieren und was dagegen getan werden kann. Die Betrachtungen werden, soweit es die gegenwärtigen Kenntnisse erlauben, quantitativ angestellt, und in diesem Sinne werden Aufgaben eingefügt, die die wichtigen Gesetze und die Anwendung dieser Gesetze auf praktische Verhältnisse beispielhaft erklären. Einige dieser Aufgaben sind im letzten Kapitel dieses Buches zusammengestellt. Für manche der Aufgaben sind die Lösungen gegeben, und zwar in erster Linie, um dem Techniker oder dem Wissenschaftler entgegenzukommen, der das Buch zur Information über Grundfragen der Korrosion benutzt und erkennt, daß das 
Lösen von Aufgaben dem notwendigen Verständnis der Problematik dient. Wie in jedem Ausbildungsfach, so ist auch hier das Lösen von Aufgaben für ein besseres und tieferes Verständnis der Thematik sehr vorteilhaft. Es wird vorausgesetzt, daß der Leser mit der elementaren physikalischen Chemie vertraut ist; diese Kenntnis ist auch für die Teilnahme am Korrosionslehrgang am MIT notwendig. Auch einige Grundkenntnisse in physikalischer Metallurgie sind nützlich, aber nicht notwendig.

Die Behandlung der Thematik ist gemäß der für die Darstellung des gesamten Stoffgebietes auf ein Semester beschränkten Zeit knapp. Aus diesem Grunde sind nur solche Literaturzitate gegeben, welche sich direkt auf den diskutierten Gegenstand beziehen. Allgemeine Literaturhinweise werden jeweils am Ende des Kapitels angeführt. Diese Hinweise enthalten für den interessierten Leser oft noch zusätzliche Literatur, jedoch ist in keinem Falle der Versuch gemacht worden, die gesamte Literatur zu zitieren, was auch den Rahmen dieses Buches überschritten hätte. Der an einem kompletteren Literturleitfaden interessierte Leser sollte daher auf solche Bücher wie ,The Corrosion Handbook", das vom Verfasser herausgegeben wurde, oder ,The Corrosion and Oxidation of Metals" von U. R. Evans zurückgreifen.

In solchen Fällen, wo mehr als eine Interpretation der Fakten oder mehrere Theorien gültig sind, ist versucht worden, beide Seiten darzulegen. Dabei hat der Verfasser seine eigene Anschauung so begründet und dargestellt, daß sie vom Leser erkannt werden kann. Die Korrosionsgeschwindigkeiten werden in den in den USA allgemein gebräuchlichen Diménsionen Milligramm pro Quadratdezimeter und Tag (mdd) und inch Abtragung pro Jahr (ipy) ausgedrückt. Das Vorzeichen des Elektrodenpotentials ist wegen der entgegengesetzten Abmachungen, die einerseits hauptsächlich in den Vereinigten Staaten und anderseits im Ausland eingehalten werden, immer ein wunder Punkt gewesen. Es bleibt zu hoffen, daß durch die Definition des Potentials gegenüber der Bezeichnung des Oxydationspotentials, in Úbereinstimmung mit der von Lewrs, Randall, Pitzer und Brever in „Thermodynamics“, 2. Auflage, Mc Graw. -Hill (1961) verwendeten Bezeichnung, eine Klarstellung geschaffen wurde, oder zum mindesten eine Verwechslung vermieden wird.

Der Verfasser ist seinen Freunden und Kollegen für viele Informationen, die seinen Vorlesungen zugute kamen und nun auch in dem vorliegenden Buche enthalten sind, sehr zu Dank verpflichtet. Zur Aufklärung der Korrosionsmechanismen hat in mehreren Fällen das geduldige Bestreben vieler Studenten beigetragen, die im Korrosionslaboratorium Untersuchungen anstellten und mit dem Verfasser nach geeigneten Experimenten suchten, oder die mühsam die nötigen Daten zusammentrugen.

Dank gebührt auch denen, die Teile des Manuskriptes gelesen und wertvolle Anregungen gegeben haben, darunter Carl WAGNER, Milton SteRN, W. D. Robertson, Bruce Chalmers, G. T. Paul, G. Schikorr, H. S. Campbell, C. P. Larrabee, W. W. Bradley und J. P. Pemsler; sowie denen, die die Photographien oder Abbildungen besorgten, insbesondere F. L. La QUE, M. Van Loo, J. F. SE- 
BALD und H. M. BendeER. Ein großer Teil des Manuskriptes wurde während eines in Göttingen (BRD) verbrachten Ruhesemesters geschrieben. Diese günstige Gelegenheit erbot sich durch die finanzielle Unterstiitzung durch die John-Simon-Guggenheim-Gedenkstiftung und durch die Gastfreundschaft von Dr. Carl WAGNER, dem Direktor des Max-Planck-Instituts für Physikalische Chemie.

Wenn dieses Buch die jungen Wissenschaftler und Techniker anregt, die anhaltenden Korrosionsprobleme zu meistern und die durch den Metallverschleiß verursachten sehr beträchtlichen ökonomischen Verluste sowie die erschreckend großen Materialverluste verringern zu.helfen, dann wird es den Hauptzweck im Sinne des Verfassers erfüllt haben.

Cambridge, Mass., November 1962 Herbert H. Uhlig 
